\title{
Intravascular missile: apparent retrograde course from the left ventricle
}

\author{
R K LAMB, A PAWADE, A L PRIOR \\ From North Staffordshire Royal Infirmary, Stoke-on-Trent
}

\begin{abstract}
An air gun pellet was found in the right superior pulmonary vein after penetrating the left ventricle of a 14 year old boy. This apparent retrograde movement in the left side of the heart has not been reported previously.
\end{abstract}

Gunshot injuries are known to produce intravascular missiles with the risk of subsequent embolisation. We report a case of injury with an air gun pellet, which penetrated the left ventricle and was later removed from the right superior pulmonary vein.

\section{Case report}

A 13 year old boy presented to the accident unit of the North Staffordshire Royal Infirmary after being shot with an air rifle in the left axilla. He was agitated, pale, and peripherally cold. There was peripheral cyanosis. The heart rate was 120 beats/min and the blood pressure was $80 / 40 \mathrm{~mm} \mathrm{Hg}$; the central venous pressure was not obviously raised and there were no audible cardiac murmurs. An entry wound was seen in the left mid axillary line, 5 th intercostal space. There was no evidence of an exit wound. A plain chest radiograph showed the pellet adjacent to the right hilum. There was no pneumothorax, the cardiac silhouette was globular, and the cardiothoracic ratio was 0.51 (figure). One hour later his heart rate had risen to 130 beats/min, the blood pressure had fallen to $70 / 40 \mathrm{~mm} \mathrm{Hg}$ and the central venous pressure was $13 \mathrm{~cm} \mathrm{H}_{2} \mathrm{O}$.

Exploration was performed through a median sternotomy and the tamponade caused by haemopericardium was relieved. A small hole was seen in the posterolateral aspect of the pericardium on the left side. A corresponding defect was evident in the left ventricle but no exit wound could be found. The ventricular wound was closed with $3 / 0$ Prolene. The right pleural cavity was opened and the pellet, localised within the extrapericardial superior pulmonary vein, was removed through a $3 \mathrm{~mm}$ incision directly on it.

The boy's recovery was uneventful and he was symptom free six months later. No cardiac murmurs were noted either

Address for correspondence: Mr R K Lamb, Wessex Cardiothoracic Centre, Southampton SO9 4XY.

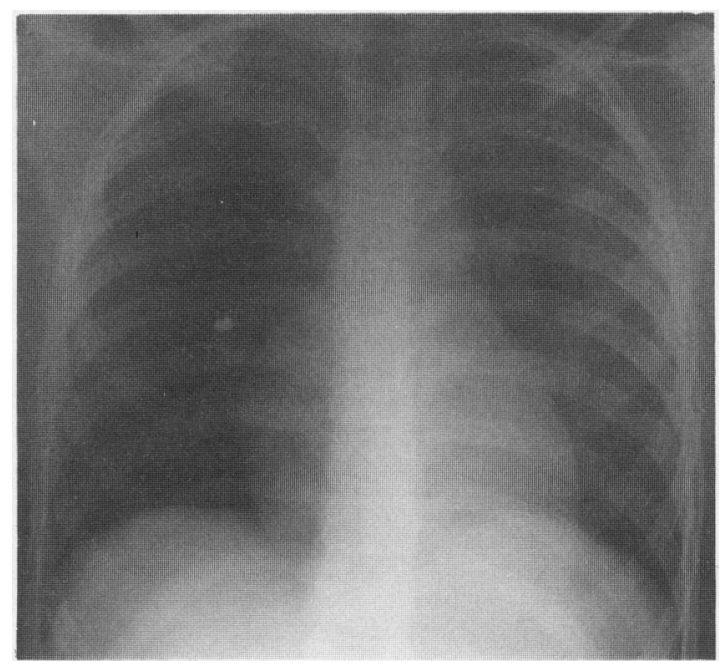

Chest radiograph (posteroanterior and lateral projections) showing a globular shaped heart and the pellet adjacent to the right hilum.

in the immediate postoperative period or when he was last seen. Subsequent chest radiographs were normal.

\section{Discussion}

Intravascular missiles are a rare sequel to gunshot injury. Bullets may gain access to the circulation via the heart and subsequent embolisation into the systemic or pulmonary vessels may occur. Antegrade migration into the pulmonary arteries results from injury to either the right atrium or the right ventricle. Similarly, penetration of the left sided chambers may produce movement into the systemic arteries. Retrograde embolisation has been reported but only in relation to the systemic venous system, pellets having entered the right atrium with subsequent migration to the hepatic and renal veins.'

The major factors affecting the fate of a bullet within the venous system include missile size, gravity, position of the body, and muscular and respiratory action. ${ }^{2}$ Movement within the systemic arteries, however, may reflect the force of blood flow, the anatomy of the arterial tree, and perhaps gravity. ${ }^{3}$ Thus embolisation into the left lower limb occurs 
more frequently than to any other site after perforation of the left ventricle. ${ }^{4}$

There is no previous report of a missile, having entered the left ventricle, being located subsequently in a pulmonary vein. For the reasons outlined above, the pellet is unlikely to have migrated into the right superior pulmonary vein after coming to rest initially in the left ventricular cavity. Rather than having moved retrogradely it is likely to have followed its original trajectory, passing across the left ventricle, mitral valve, and left atrium before coming to a halt in the pulmonary vein. The relatively low kinetic energy of this projectile $(\cdot 22)$ prevented further penetration.

Missiles may perforate the heart and the patient present without cardiac tamponade. ${ }^{2}$ These patients remain without symptoms until the bullet is seen, either before or after embolisation, on a plain radiograph. ${ }^{4}$ In the former case its exact location could be established by either computed tomography or echocardiography. Such investigations would seem reasonable when either intravascular or intracar- diac penetration is thought likely as the possibility subsequent embolisation cannot be ignored.

This case illustrates the need to confirm the presence of a exit wound when the heart is perforated by a missile. Shoula only the entry site be visible, the surgeon must be aware of the possibility of both antegrade and retrograde embolisation even within the left heart.

\section{References}

I Mattox KL, Beall AC, Ennix CL, et al. Intravascular migrato bullets. Am J Surg 1979;137:192-5.

2 Trimble C. Arterial bullet embolism following thoracic gunsho wounds. Ann Surg 1968;168:911-6.

3 Garzon A, Gliedman ML. Peripheral embolization of a bulli following perforation of the thoracic aorta. Ann Su㐘 1964;160:901-3.

4 Symbas PN, Harlaftis N. Bullet emboli in the pulmonary and systemic arteries. Ann Surg 1977;185:318-20. 